\title{
BAER SUBPLANES AND BLOCKING SETS
}

\author{
BY A. BRUEN
}

Communicated by H. S. M. Coxeter, September 19, 1969

A blocking set $S$ in a projective plane $\pi$ is a subset of the points of $\pi$ such that every line of $\pi$ contains at least one point of $S$ and at least one point which is not in $S$. Denoting the number of points in $S$ by $|S|$ our main result, obtained by purely combinatorial means, is the following: If $\pi$ is finite of square order, say $m^{2}$ then $|S| \geqq m^{2}+m$ +1 and if $|S|=m^{2}+m+1$ then the points of $S$ are the points of a subplane of $\pi$ of order $m$ (a Baer subplane). In this connection we first of all prove the following

THEOREM. Baer subplanes form blocking sets.

Proof. Suppose $\pi$ is a plane of order $m^{2}$ which contains a subplane $S$ of order $m$. Since any line of $\pi$ contains at most $m+1$ points of $S$ we have that every line of $\pi$ contains at least one point which is not in $S$. Let $l$ be any line of $\pi$ and $P$ be any point of $l$ which is not in $S$. Then there is at most one line of $S$ through $P, S$ being a subplane. Also since any two points of $\pi$ are connected by a unique line, the $m^{2}+m+1$ points of $S$ are contained in the $m^{2}+1$ lines of $\pi$ through $P$. If $l$ contained no point of $S$, the lines of $\pi$ through $P$ would account for at most $(m+1)+\left(m^{2}-1\right) \cdot 1=m^{2}+m$ points of $S$. Thus $l$ must contain at least one point of $S$ establishing our theorem.

We now proceed to the main result. $\pi$ denotes a plane of order $n$ and $S$ is a blocking set in $\pi . S-l$ denotes all those points $P$ such that $P$ is contained in $S$ but not in $l$, and $|S-l|$ means the number of such points $P$; similarly for $l-S,|l-S|$.

Lemma 1. No line of $\pi$ contains more than $|S|-n$ points of $S$.

Proof. Let $l$ be any line of $\pi$ and suppose $l$ contains exactly $t$ points of $S$. Since $S$ is a blocking set there is at least one point $R$ in $l-S$. There are $n$ lines of $\pi$ through $R$ besides $l$, each containing at least one point of $S$. Thus always $|S| \geqq t+n$.

Lemma 2. Let a objects be packed into $b$ boxes such that each box contains at least one object, with $b \leqq a<2 b$. Define a function $f$ on the objects $X$ as follows:

AMS Subject Classifications. Primary 5070, 5060; Secondary 0527.

Key Words and Phrases. Finite projective plane, Baer subplane, incidences, blocking sets. 
$f(X)=1$ if the box containing $X$ contains other objects, $f(X)=0$ otherwise.

Then for any such packing $P$, we have

$$
A(P)=\sum f(X) \leqq 2(a-b),
$$

the summation being over all objects $X$.

Proof. It can be seen that if some box contains more than two objects, for some packing $P$, there is a packing $P^{\prime}$ such that $A\left(P^{\prime}\right)$ $>A(P)$. Hence $A(P)$ attains its maximum value when each box contains no more than two objects, and, in this case, $A(P)=2(a-b)$.

From now on we assume that $n$ is a square, $n=m^{2}$, say.

Lemma 3. Suppose $|S|=m^{2}+m+1$. Let some line $l$ of $\pi$ contain exactly $k$ points of $S$. Let $B$ denote those lines of $\pi$ passing through points of $l-S$ and containing at least two points of $S-l$. Let $I$ denote the set of incidences of points of $S-l$ with lines of $B$. Then $|I| \leqq 2(m+1-k)$ $m^{2}+1-k$.

Proof. For each point $P$ in $l-S$ the $m^{2}+m+1-k$ points of $S-l$ are packed into $m^{2}$ lines through $P$. Hence, by Lemma 2 these lines through $P$ yield at most $2\left[\left(m^{2}+m+1-k\right)-m^{2}\right]$ incidences in I. Thus, since $|l-S|=m^{2}+1-k$, we have

$$
|I| \leqq 2\left(m^{2}+1-k\right)(m+1-k) \text {. }
$$

LEMMA 4. If $|S|=m^{2}+m+1$, some line of $\pi$ contains precisely $m+1$ points of $S$.

Proof. Let some line $l$ of $\pi$ contain precisely $k$ points of $S$ where $k$ is the maximum number of points of $S$ on any line of $\pi$. Clearly $k \geqq 2$ and, by Lemma $1, k \leqq m+1$. Let $B, I$ be as in Lemma 3 , and $P$ any point of $S-l$. There remain $m^{2}+m-k$ points of $S-l$ and the $k$ lines of $\pi$ which connect $P$ to points of $S \cap l$ account for at most $k(k-2)$ of them. Thus there are at least $m^{2}+m-k-k(k-2)$ points of $S-l$ different from $P$ and also incident with lines of $B$ through $P$. If there are $b$ lines of $B$ through $P$ we must have $b(k-1) \geqq\left[m^{2}+m-k\right.$ $-k(k-2)]$. Thus the lines of $B$ through $P$ yield at least $b$ incidences in $I$, where $b \geqq(m+1-k)(m+k)(k-1)^{-1}$. Summing over all the points of $S-l$ such as $P$ we obtain $|I| \geqq\left(m^{2}+m+1-k\right) b$. Thus, from Lemma 3, we must have

$$
2\left(m^{2}+1-k\right)(m+1-k) \geqq\left(m^{2}+m+1-k\right) b .
$$

If we assume $k<m+1$ we have $2(k-1)\left(m^{2}+1-k\right) \geqq\left(m^{2}+m+1-k\right)$ 
$(m+k)$. Now, $k \leqq m \Rightarrow 2(k-1)<2 k \leqq(m+k)$ and $m^{2}+1-k<m^{2}+m$ $+1-k$, that is, the supposition $k<m+1$ is contradictory. Thus, from Lemma $1, k=m+1$, and some line of $\pi$ contains precisely $m+1$ points of $S$.

Theorem 1. If $|S|=m^{2}+m+1$, then the points of $S$ are the points of a Baer subplane of $\pi$.

Proof. By Lemma 4 some line $l$ of $\pi$ contains precisely $m+1$ points of $S$. Since $S$ is a blocking set, we have that if $U$ and $V$ are any two distinct points of $S-l$ the line $U V$ of $\pi$ must meet $l$ in a point of $S \cap l$. Thus for any point $P$ of $S-l$ the $(m+1)$ lines of $\pi$ connecting $P$ to the $m+1$ points of $S \cap l$ account for all the $m^{2}$ points of $S-l$, and, using Lemma 1 , each such line contains precisely $m+1$ points of $S$. Hence if we define a structure $\pi^{\prime}$ such that the points of $\pi^{\prime}$ are the points of $S$, the lines of $\pi^{\prime}$ are those lines of $\pi$ containing at least two points of $S$, and incidence in $\pi^{\prime}$ is given by incidence in $\pi$, it can be seen that $\pi^{\prime}$ is a subplane of $\pi$, and $\pi^{\prime}$ has order $m$.

Theorem 2. $|S| \geqq m^{2}+m+1$.

Suppose $|S|=m^{2}+m+1-t, t>0$. By Lemma 1 no line of $\pi$ contains more than $m+1-t$ points of $S$. Let $L$ be any set of $t$ points of $\pi$ none of which is in $S$ and such that the points of $S^{\prime}$ do not form the points of a Baer subplane of $\pi$ where $S^{\prime}=S \cup L$. Then $S^{\prime}$ is a blocking set since no line of $\pi$ contains more than $(m+1-t)+t$ points of $S^{\prime}$. Thus we would have a blocking set $S^{\prime}$ with $\left(S^{\prime}\right)=m^{2}+m+1$; by the condition on $L$ this contradicts Theorem 1 .

REMARK. The author has since proved that $|S| \geqq n+n^{1 / 2}+1$ for $\pi$ of order $n, n$ arbitrary. This result and some corollaries will be discussed elsewhere.

Acrnowledgement. I wish to thank sincerely C. Fisher and my supervisor Professor F. A. Sherk of Toronto for their valuable help and encouragement in connection with this work.

University of Missouri, Columbia, Missouri 65201 